\title{
Relato de experiência da terapia fonoaudiológica com enfoque vocal no grupo de Parkinson - NETI/APASC
}

\author{
Maria Rita Pimenta Rolim \\ rita.pimenta@ufsc.br
}

\section{Resumo}

O trabalho do projeto de extensão universitária denominado "Terapia Vocal em Portadores de Parkinson" é desenvolvido juntamente com o Programa Grupo de Apoio aos Portadores da Doença de Parkinson e seus Familiares, no Núcleo de Estudos da Terceira Idade da Universidade Federal de Santa Catarina NETI/UFSC e a APASC. O Grupo t Apoio tem a concepção de uma estratégia de cuidado grupal, integrando pessoas que compartilham situações de vida similares, constituindo-se em um programa com significativo impacto no processo de viver de seus integrantes, pois trata-se de um espaço apoiado em relações de confiança que possibilita a livre expressão de sentimentos e o compartilhamento de vivências, informações sobre a doença e estratégias de cuidado, promovendo o apoio para o enfrentamento de perdas progressivas e li-

mitações impostas pelo problema vivenciado, favorecendo a autonomia e independência, e o empoderamento das pessoas, resultando na manutenção ou na criação de novos vínculos sociais. Nesse grupo são oferecidos projetos de extensão nas áreas de educação física, fonoaudiologia, cognição, fisioterapia, nos quais os portadores de DP podem se inserir. O grupo de portadores de DP participantes do projeto é formado por $76 \%$ do sexo masculino e $24 \%$ do sexo feminino, sendo alguns acompanhados de seus familiares e/ou cuidadores. As estratégias de intervenção utilizadas neste projeto baseiam-se em técnicas e procedimentos que visam trabalhar as áreas da comunicação, expressão corporal e facial.

Palavras-chave: Voz. Parkinson. Qualidade de vida.

\section{Experience report of speech therapy focused vocal group Parkinson - NETI/APASC}

\begin{abstract}
The work of the extension project called "Vocal Therapy in Patients with Parkinson" is developed along with the Program Support Group for People with Parkinson's Disease and their Families, the Center for Studies of the Elderly, Federal University of Santa Catarina NETI / UFSC and APASC. The Support Group is designing a care strategy group, integrating people who share similar life situations, being in a program with a significant impact on the process of living of its members, because it is a space for relationships supported of trust that

allows the free expression of feelings and share experiences, information about the disease and care strategies, promoting support for coping with progressive losses and limitations imposed by the problem experienced, favoring autonomy and independence, and empowerment of people, resulting
\end{abstract}




\section{Extensio $\mid$ Relato de Experiência}

in the maintenance or creation of new social ties. In this group are offered extension projects in the areas of physical education, speech, cognition, physical therapy, in which patients with PD may be inserted. The group of participants with PD project consists of $76 \%$ male and $24 \%$ female, with some accompanied by their families and / or caregivers. Intervention strategies used in this project are based on techniques and procedures to work the fields of communication, body language and facial.

Keywords: Voice. Parkinson. Quality of Life.

\section{Introdução}

As alterações demográficas do último século, que se traduziram na modificação e, por vezes, na inversão das pirâmides etárias, refletiram no envelhecimento da população com saúde, autonomia e independência, o maior tempo possível, constituindo, assim, um desafio à responsabilidade individual e coletiva.

Com o envelhecimento, todos os indivíduos saudáveis apresentam morte progressiva das células nervosas que produzem dopamina. Algumas pessoas, entretanto, perdem essas células (e consequentemente diminuem muito mais seus níveis de dopamina) num ritmo muito acelerado e, assim, acabam por manifestar os sintomas da doença de Parkinson (DP). Não se sabe exatamente quais são os motivos que levam a essa perda progressiva e exagerada de células nervosas (degeneração), muito embora o empenho de estudiosos desse assunto seja muito grande (MENESES,TEIVE, 1996).

A doença de Parkinson é uma enfermidade descrita pela primeira vez em 1817, pelo médico inglês James Parkinson: "Doença neurológica progressiva que afeta os centros cerebrais responsáveis pelo controle e regulação do movimento" (REIS, 2004). É um distúrbio neurológico degenerativo-progressivo, que afeta o bulbo olfatório e o nervo vago, caracterizado principalmente pela degeneração das células (neurônios) da camada ventral da parte compacta da substância negra e do lócus coeruleus. Tal degeneração resulta na diminuição da produção de dopamina, produzindo um conjunto de sintomas caracterizados principalmente por distúrbios motores. Seu início costuma ser insidioso, e dificilmente o portador identifica o momento exato em que notou alguma mudança em si; geralmente são parentes ou pessoas próximas que percebem alterações sutis (MENESES; TEIVE, 1996). A incapacidade produzida pelos sintomas motores da doença caracterizase pelos principais sinais da doença, que são: presença de tremor de repouso (sobretudo das mãos); rigidez muscular; bradicinesias, que se traduzem por lentidão dos movimentos e dificuldade em iniciar movimentos voluntários; instabilidade postural por perda de reflexos posturais; além de alterações de voz e fala (LIMONGI, 2001). Entre toda a gama de sinais e sintomas que a configuram, distúrbios na área de comunicação envolvendo alterações de fonação e deglutição acometem $75 \%$ dos casos de forma significativa (CALDAS, 2004).

Os casos de demência são comumente observados em pacientes com DP avançada, principalmente em idosos, embora tal fato não seja inteiramente compreendido. E ainda: uma proporção considerável de casos, podendo chegar a 40\%, apresenta depressão em graus variados, que se manifesta nos pacientes por uma queda na motivação para exercícios físicos e atividades associativas e na iniciativa para inovações. Além disso, podem apresentar sintomas como melancolia, perda de apetite, fadiga, distúrbios do sono, perda da autoestima e ansiedade (LIMONGI, 2001; REIS, 2004).

A doença de Parkinson ocorre em todo o mundo, afetando pessoas de ambos os sexos, independente de raça ou classe social. Predomina em pessoas idosas, com início do quadro clínico geralmente entre os 50 e 70 anos de idade, embora não seja rara a incidência mais precoce (LIMONGI, 2001; CALDAS, 2004; REIS, 2004). A sua prevalência aumenta de $0,6 \%$, aos 65 anos, para 3,5\%, aos 85 e mais anos; no mundo inteiro, são mais de dez milhões de pacientes. No Brasil, o número de portadores deve beirar os 300 mil indivíduos, sendo uma das doenças crônicas neurodegenerativas mais comuns na população idosa (PORTAL DA SAÚDE, 2011). 
Quanto ao tratamento, ainda não existem medicamentos capazes de interromper o curso da doença, nem de evitá-la; os existentes visam ao controle dos sintomas com o objetivo de manter o portador com autonomia, independência funcional e equilíbrio psicológico, o que se obtém com a reposição de dopamina estriatal. A administração de levodopa é a terapia medicamentosa mais recomendada no controle satisfatório dos sintomas (LIMONGI, 2001).

Remédios não são os únicos recursos para combater os sintomas; são de fundamental importância: os cuidados de enfermagem, de educação física, de fisioterapia, fonoaudiologia e terapia ocupacional, entre outros, desenvolvidos tanto individualmente quanto em grupo, para restituição possível da capacidade funcional, do bem-estar e da qualidade de vida (CALDAS, 2004).

\section{Núcleo de Estudos da Terceira Idade da Universidade Federal de Santa Catarina (NETI/UFSC)}

Criado pela Portaria 484/GR/83, o Núcleo de Estudos da Terceira Idade da Universidade Federal de Santa Catarina NETI/UFSC tem por objetivo a elaboração, sistematização, socialização e ampliação de conhecimentos de gerontologia, baseados no planejamento, desenvolvimento e avaliação de ações que, em consonância com a realidade universitária e comunitária, possam integrar a população idosa ao meio acadêmico e comunitário, como sujeitos em transformação e transformadores, tendo em vista o estabelecimento de uma política de resgate do papel do idoso na sociedade brasileira.

É no NETI que é realizado o Grupo de Apoio aos Portadores da Doença de Parkinson, que é um encontro quinzenal destinado aos portadores e seus familiares e tem o objetivo de apoiá-los para enfrentarem a doença e fortalecerem sua cidadania enquanto usuários de serviço de saúde, acerca de suas reivindicações e controle social.

\section{Associação Parkinson de Santa Catarina (APASC)}

A Associação Parkinson de Santa Catarina foi fundada em 31 de março de 2004, com a finalidade de congregar, apoiar e defender os direitos de seus associados.

A APASC articula com entidades da comunidade atividades aos seus associados visando a uma melhor qualidade de vida aos portadores de Parkinson. É nesse contexto que o Curso de Fonoaudiologia da UFSC está inserido neste projeto.

\section{Qualidade de vida e promoção de saúde}

Segundo a Organização Mundial de Saúde (OMS) (PORTAL DA SAÚDE, 2011), saúde é definida como "[...] estado de completo bem-estar físico, mental e social e não somente pela ausência de doença ou enfermidade." O conceito de qualidade de vida relacionada à saúde (QVRS) é bastante complexo, e, em geral, saúde é aceita como parte essencial da QV que engloba um conceito multidimensional que reflete a avaliação subjetiva de satisfação pessoal em relação ao bem-estar físico, funcional, emocional e social (PASCHOAL, 2001). Nesse sentido, deve-se considerar que o foco mais importante da qualidade de vida no âmbito da saúde é o conceito de promoção de saúde, centrada na capacidade de viver sem doenças ou de superar as dificuldades dos estados ou concepções de morbidade (MINAYO; HARTZ; BUSS, 2000).

Do ponto de vista da coletividade, sendo o envelhecimento um fenômeno que diz respeito a todos os seres humanos, implica necessariamente todos os setores sociais, exigindo a sua intervenção e corresponsabilização na promoção da autonomia e da independência das pessoas idosas e o envolvimento das famílias e de outros 
prestadores de cuidados profissionais (ALVAREZ, SCHNEIDER, GONALVES, 2002).

Considera-se necessário que o planejamento do cuidado seja um instrumento de atenção à saúde que apresente grande potencial de construção de um plano de cuidado integral e humanístico. Assim, esse plano de cuidado deve ser desenvolvido de forma a favorecer o cuidado integral e contínuo para a melhora da qualidade de vida. Torna-se fundamental um processo de avaliação contínuo para intervir e adequar as novas estratégias de intervenção quando necessário (ALVAREZ; SCHNEIDER; GONALVES, 2002).

Diante disso, a necessidade prática tem demonstrado, pelas muitas experiências positivas de grupos de ajuda mútua que prestam apoio psicossocial às pessoas que passam por eventos traumáticos e estressantes, que esse tipo de organização grupal é um recurso valioso e pouco oneroso na preservação da saúde da coletividade que vivencia eventos de vida de difícil enfrentamento (GONALVES; ALVAREZ; ARRUDA, 2007).

Nessa perspectiva, o trabalho do projeto de extensão universitária denominado "Terapia Vocal em Portadores de Parkinson" (protocolo de aprovação n. 2011.3635) é desenvolvido juntamente com o Programa Grupo de Apoio aos Portadores da Doença de Parkinson e seus Familiares, no Núcleo de Estudos da Terceira Idade da Universidade Federal de Santa Catarina NETI/UFSC e a APASC.

O Grupo de Apoio tem a concepção de uma estratégia de cuidado grupal, integrando pessoas que compartilham situações de vida similares, constituindo-se em um programa com significativo impacto no processo de viver de seus integrantes, pois trata-se de um espaço apoiado em relações de confiança que possibilita a livre expressão de sentimentos e o compartilhamento de vivências, informações sobre a doença e estratégias de cuidado, promovendo o apoio para o enfrentamento de perdas progressivas e limitações impostas pelo problema vivenciado, favorecendo a autonomia e independência, e o empoderamento das pessoas, resultando na manutenção ou na criação de novos vínculos sociais. Nesse grupo são oferecidos projetos de extensão nas áreas de educação física, fonoaudiologia, cognição, fisioterapia, nos quais os portadores de DP podem se inserir.

\section{Terapia Vocal em Portadores de Parkinson}

Alterações da qualidade da voz, fala e até mesmo linguagem, além das dificuldades de deglutição, são sintomas importantes observados na DP, prejudicando a comunicação oral, pois todo o mecanismo fonatório na DP encontra-se afetado, uma vez que funções neuromusculares são necessárias para produção de fala inteligível (RAMIG, 2001; BEHLAU, 2001).

As alterações da voz e da fala na DP constituem, em conjunto, o que se denomina disartria hipocinética, ou disartrofonia, e caracterizam-se por monotonia e redução da intensidade da voz, articulação imprecisa e distúrbios do ritmo; tais problemas de comunicação podem favorecer o isolamento social (BEHLAU, 2001). Os distúrbios da voz decorrem de três fatores principais: restrições na modulação da frequência e intensidade, redução da intensidade e alterações da qualidade. Por sua vez, os distúrbios da articulação resultam em imprecisão na emissão de consoantes e decorrem da redução dos movimentos dos lábios e da língua em seus diversos pontos e modos de articulação. Comprometimento da coordenação dos movimentos respiratórios e das funções de ressonância, embora presente em graus variáveis, não resulta em piora da inteligibilidade da fala (RAMIG, 2001; BEHLAU, 2001). De acordo com estudos recentes, o fator decisivo para a redução da inteligibilidade da comunicação oral na DP é a redução da intensidade vocal (COUTINHO, 2009).

Dessa forma, muitos pacientes experimentam piora gradual de sua capacidade de comunicação à medida que a doença progride, pois, frequentemente, o tratamento farmacológico é menos eficaz no que diz respeito à disartrofonia quando comparado com outras manifestações clínicas (RAMIG, SAPIR, FOX, COUNTTRYMAN, 2001).

Tradicionalmente, o tratamento fonoaudiológico para as alterações vocais do indivíduo parkinsoniano envolve três abordagens distintas: mioterapia, coordenação das estruturas de fala e respiração (GASPARINI; DIAFÉRIA; BEHLAU, 2003). Geralmente é realizado, de uma a duas vezes por semana, enfatizando a articulação, velocidade e prosódia. A literatura mostra evidências de nível I para o método de reabilitação Lee 
Silverman VoiceTreatment (LSVT'), desenvolvido por Ramig e Col (2001), que enfoca o nível laríngeo para o tratamento dos desvios de voz e fala de indivíduos com DP.

A partir da perspectiva de promoção de saúde vocal, foram traçados objetivos específicos para a melhoria da qualidade de vida dos integrantes do grupo de portadores da DP no intuito de melhorar a sua comunicação e readquirir parte de sua autonomia dentro das limitações impostas pela doença, além de capacidade de enfrentamento dela, que envolvem: identificar as necessidades individuais e coletivas; estabelecer um plano terapêutico intervindo sobre as necessidades identificadas; reavaliar as intervenções e readequá-las conforme as necessidades identificadas.

Baseado no princípio fundamental de que a responsabilidade com a saúde estende-se durante toda a vida, buscando melhorias, condições e qualidade de vida, e a partir de um olhar ampliado, sem restringir-se apenas à sua patologia e promovendo ao mesmo tempo a participação ativa dos integrantes do grupo, propõese um modo diferenciado de ação, através do atendimento em grupo pela musicoterapia e fonoaudiologia de um grupo de portadores de DP.

Nesse sentido, também contribuindo para a atuação sobre os determinantes da perda de autonomia e de independência, objetiva-se realizar um trabalho numa visão integradora, focando na comunicação objetiva dos portadores de DP, através da convivência em grupo, estimulando a produção individual para um convívio social adequado.

\section{População-Alvo}

O grupo de portadores de DP participantes do projeto é formado por $76 \%$ do sexo masculino e $24 \%$ do sexo feminino, sendo alguns acompanhados de seus familiares e/ou cuidadores.

\section{Estratégias de Intervenção}

As estratégias de intervenção utilizadas neste projeto baseiam-se em técnicas e procedimentos que visam trabalhar as áreas da comunicação, expressão corporal e facial.

Inicialmente realizou-se a Avaliação do Perfil de Extensão Vocal com o software Vocalgrama de CTS Informática. A avaliação desse programa consiste na contagem numérica em quatro situações de fala. Iniciouse com a fala habitual (média), depois com voz fraca, forte e muito forte. A cada semestre de intervenção, foi feita uma reavaliação utilizando o mesmo software.

O programa foi realizado em 2010, inicialmente com encontros semanais, com duração de uma hora e meia, na Associação Comunitária do Jardim Santa Mônica (ACOJAR), em Florianópolis/SC. A partir de 2011, os encontros passaram a ocorrer na sede do NETI/sala da APASC e sempre tiveram a participação de alunos do curso de Fonoaudiologia, bolsistas da Pró-Reitoria de Assuntos Estudantis (PRAE) da Universidade Federal de Santa Catarina (UFSC), da professora do Curso de Fonoaudiologia da UFSC, coordenadora do projeto de Terapia Vocal e uma musicoterapeuta voluntária no projeto.

Todos os encontros iniciaram com exercícios que visam à propriocepção corporal com enfoque na região cervical associados à coordenação fonorespiratória, dessa forma focalizando o nível laríngeo com emissões de sons facilitadores. Após, foram inseridos exercícios miofuncionais, isotônicos e isométricos, objetivando a melhoria da expressão facial e articulação dos sons da fala. No segmento, são sugeridos exercícios específicos da função vocal. Exercícios esses que visam aumentar a intensidade vocal, aprimorar a precisão articulatória em sequência e equilibrar a ressonância.

Como parte final do encontro, por intermédio da música, promoveu-se o prazer na atividade, estimulouse a comunicação e o ritmo, trabalhou-se a respiração, a melhora na qualidade vocal e a expressão integral, a atenção e a memorização, ajudando a automatizar os novos comportamentos. 
Todas as atividades propostas no grupo desenvolvem a inter-relação pessoal, contribuindo na integração social, com apresentações em público (SEPEX/UFSC, Festas de Final de Ano, VI Congresso Brasileiro das Associações de Parkinson do Brasil) do repertório musical trabalhado.

\section{Considerações finais}

O grupo de Parkinson, além de corresponder a um novo espaço de identificação de suporte social para os portadores e seus familiares, favorece ainda a constituição de uma rede ampliada útil às demandas do grupo na perspectiva da integralidade da pessoa.

A relação interpessoal do grupo permite afirmar que há maior qualidade final deste trabalho. Salienta-se que os participantes obtiveram melhoras nas suas desempenhos vocais, melhor fixação dos parâmetros vocais trabalhados através da música, o que proporcionou uma interação entre eles e os alunos que participam do grupo. Percebeu-se a alegria e a assiduidade com que chegam ao encontro, o prazer em executar as atividades propostas e, segundo relatos, melhora na comunicação em geral e na autoestima; bem como o compartilhar de suas necessidades e conquistas com o grupo, o que eleva a qualidade de vida dos portadores e seus familiares.

A realização desse trabalho em grupo demonstra ser de grande valia, pois proporciona o exercício da gestão do cuidado, a valorização do trabalho em equipe e a complexidade de desenvolver cuidados individuais e coletivos.

Torna-se relevante destacar a afirmação de Matumoto (1998), na qual relata que:

[...] compreender melhor a relação entre pessoas portadoras de um agravo poderia ajudar a elaborar melhor as estratégias para o resgate do humano, da cidadania e da vida na saúde. Na posição de trabalhadores de saúde temos a responsabilidade social de lidar com necessidades, dores e sofrimentos do outro. É essencial nos questionar constantemente no dia a dia da nossa prática sobre esse outro com quem me relaciono: Quem é esse outro? Como me relaciono com ele? Para quê?

Considera-se importante ressaltar que essa atividade em grupo é realizada continuamente, sendo renovado o projeto a cada ano.

Acredita-se que esse exercício em grupo possa vir a criar espaços de mudanças na prática de saúde, reforçando a prática da humanização do cuidado, essência da atuação dos profissionais de saúde.

\section{Referências}

ALVAREZ, A. M. Tendo que cuidar: a vivência do idoso e de sua família cuidadora no processo de cuidar e ser cuidado em contexto domiciliar. 2011. Dissertação (Pós-Graduação em Enfermagem) - Programa de PósGraduação em Enfermagem, Universidade Federal de Santa Catarina, Florianópolis, 2001.

ALVAREZ, A. M.; SCHNEIDER P.; GONALVES, L. H. T. Grupo de ajuda mútua de familiares de idosos portadores de Doença de Alzheimer e doenças similares do HU/UFSC. Rev. Ciênc. Saúde, v. 21, n.2, p. 54-66, 2002.

BEHLAU, M. Voz: o livro do especialista. Rio de Janeiro: Revinter, 2001: 265-266.

CALDAS, C.P. A saúde do idoso: a arte de cuidar. Rio de Janeiro: EdUERJ; 2004.

COUTINHO, Sylvia Boechat et al . Voz e fala de Parkinsonianos durante situações de amplificação, atraso e 
mascaramento. Pró-Fono R. Atual. Cient., Barueri, v. 21, n. 3, Set. 2009 . Disponível em: <http://www. scielo.br/scielo.php?script=sci_arttext\&pid=S0104-56872009000300007\&lng=en\&nrm=iso >. Accesso em: mai. 2013.

GASPARINI, G.; DIAFÉRIA, G.; BEHLAU, M. Queixa vocal e análise perceptivo-auditiva de pacientes com doença de Parkinson. R. Ci. méd. biol., Salvador, v. 2, n. 1, p. 72-76, jan./jun. 2003

GONÇAlVES, L.H.T; ALVAREZ, A.M.; ARRUDA, M.C. Pacientes portadores da doença de Parkinson: significado de suas vivências. Acta Paul Enferm, v.20, n.1, p. 62-80, 2007. Disponível em: < http://www. gerontologia.org/noticia.php?id=206>. Acesso em: 10 out.2011.

LEITE, M. C. P. Doença de Parkinson. Informedicals Policlin. 2005. Disponível em: http://www. doencadeparkinson.com.br. Acesso em: 8 set. 2011.

LIMONGI, J.C.P., (ORG). Conhecendo melhor a Doença de Parkinson: uma abordagem multidisciplinar com orientações práticas para o dia-a-dia. São Paulo: Plexus; 2001.

MATUMOTO, S. O acolhimento: um estudo sobre seus componentes e sua produção em uma unidade da rede básica de serviços de saúde. 1998. Dissertação (Mestrado em Enfermagem) - Escola de Enfermagem de Ribeirão Preto, Universidade do Estado de São Paulo, Ribeirão Preto, 1998.

MENESES, M.S.; TEIVE, H.A.G. Doença de Parkinson: aspectos clínicos e cirúrgicos. Rio de Janeiro: Guanabara-Koogan;1996.

MINAYO, M.C.S.; HARTZ, Z.M.A.; BUSS, P.M. Qualidade de vida e saúde: um debate necessário. Ciên. Saúde Coletiva, v.5, n.1, p.7-18, 2000.

PASCHOAL, S.M.P. Qualidade de vida no idoso: elaboração de um instrumento que privilegia sua opinião. 2001. Dissertação (Mestrado em Saúde Pública) Faculdade de Saúde Pública, Universidade de São Paulo, São Paulo, 2001.

RAMIG, L.O. et al. Intensive voice treatment (LSVT) for patients with Parkinson's disease: a 2 year follow up. J Neurol Neurosurg Psychiatry, v.71, p.493-498, 2001.

RAMIG, L.O.; SAPIR S.; FOX C. COUNTRYMAN, S. Changes in vocal loudness following intensive voice treatment (LSVT) in individuals with Parkinson's disease: a comparison with untreated patients and normal age-matched controls. Mov Disord 2001;16:79-83.

REIS, T. Doença de Parkinson: pacientes, familiares e cuidadores. Porto Alegre: Pallotti; 2004. 